\title{
Characterization of SSR genomic abundance and identification of SSR markers for population genetics in Chinese jujube (Ziziphus jujuba Mill.)
}

Peng-cheng Fu, Yan-Zhao Zhang, Hui-yuan Ya, Qing-bo Gao

Chinese jujube (Ziziphus jujuba Mill. [Rhamnaceae]), native to China, is a major dried fruit crop in Asia. Although many simple sequence repeat (SSR) markers are available for phylogenetic analysis of jujube cultivars, few of these are validated on the level of jujube populations. In this study, we first examined the abundance of jujube SSRs with repeated unit lengths of 1-6 base pairs, and compared their distribution with those in Arabidopsis thaliana. We identified 280,596 SSRs in the assembled genome of jujube. The density of SSRs in jujube was 872.60 loci/Mb, which was much higher than in $A$. thaliana (221.78 loci/Mb). (A+T)-rich repeats were dominant in the jujube genome. We then randomly selected 100 SSRs in the jujube genome with long repeats and used them to successfully design 70 primer pairs. After screening using a series of criteria, a set of 20 fluorescently labeled primer pairs was further selected and screened for polymorphisms among three jujube populations. The average number of alleles per locus was 12.8. Among the three populations, mean observed and expected heterozygosities ranged from 0.858 to 0.967 and 0.578 to 0.844 , respectively. After testing in three populations, all SSRs loci were in Hardy-Weinberg equilibrium (HWE) in at least one population. Finally, removing high null allele frequency loci and linked loci, a set of 17 unlinked loci was in HWE. These markers will facilitate the study of jujube genetic structure and help elucidate the evolutionary history of this important fruit crop. 
1 Characterization of SSR genomic abundance and identification of SSR

2 markers for population genetics in Chinese jujube (Ziziphus jujuba

Mill.)

Peng-cheng Fu' ${ }^{1}$, Yan-zhao Zhang ${ }^{1}$, Hui-yuan Ya', Qing-bo Gao ${ }^{2}$

5

$6{ }^{1}$ College of Life Science, Luoyang Normal University, Luoyang, Henan, China

$7 \quad{ }^{2}$ Key Laboratory of Adaptation and Evolution of Plateau Biota, Northwest Institute of

8 Plateau Biology, Chinese Academy of Sciences, Xining, Qinghai, China

Corresponding author:

Qing-bo Gao

No. 59 Xiguan Avenue, Xining 810001, Qinghai, China

E-mail address: qbgao@nwipb.cas.cn

\section{Abstract}

Chinese jujube (Ziziphus jujuba Mill. [Rhamnaceae]), native to China, is a major dried fruit crop in Asia. Although many simple sequence repeat (SSR) markers are available for phylogenetic analysis of jujube cultivars, few of these are validated on the level of jujube populations. In this study, we first examined the abundance of jujube SSRs with repeated unit lengths of 1-6 base pairs, and compared their distribution with those in Arabidopsis thaliana. We identified 280,596 SSRs in the assembled genome of jujube. The density of SSRs in jujube was 872.60 loci/Mb, which was much higher than in A. thaliana $(221.78$ loci/Mb). (A+T)-rich repeats were dominant in the jujube genome. We then randomly selected 100 SSRs in the jujube genome with long criteria, a set of 20 fluorescently labeled primer pairs was further selected and screened for polymorphisms among three jujube populations. The average number of alleles per locus was 12.8. Among the three populations, mean observed and expected heterozygosities ranged from 0.858 to 0.967 and 0.578 to 0.844 , respectively. After testing in three populations, all SSRs loci were in Hardy-Weinberg equilibrium (HWE) in at least one population. Finally, removing high null allele frequency loci and linked loci, a set of 17 unlinked loci was in HWE. These markers will facilitate the study of jujube genetic structure and help elucidate the evolutionary history of this important fruit crop. 


\section{INTRODUCTION}

Chinese jujube (Ziziphus jujuba Mill.; $2 n=2 x=24$ ) is an economically important species in the Rhamnaceae that is native to China (Chen \& Schirarend, 2007). One of the world's oldest cultivated fruit tree crops, according to archaeological evidence jujube was used at least 7,700 years ago in China (Liu \& Zhao, 2009). Fruits of jujube have high nutritional value as well as medicinal properties, and are consumed fresh, dried or in processed form (Li et al., 2007).

Over a long period of natural evolution and artificial selection, Chinese jujube has acquired a wide range of variation, with more than 800 cultivars reported (Liu et al., 2009). These cultivars are distributed across China and are propagated either by rootstock grafting or as rooted cuttings (Wang, 2001). The origins of most of these cultivars are obscure because of the frequent exchange of plant material between different cultivation areas and the lack of cultivar historical documentation (Wang et al., 2014). During the frequent exchanges, the gene flow among cultivars and the gene flow between cultivars and their wild relatives are not reported. The evolutionary history of jujube cultivars thus remains unclear.

However, genetic studies based on molecular markers could offer new clues to the evolutionary history of cultivars (Sefc et al., 2000; Testolin et al., 2000; Pan et al., 2011). Simple sequence repeats (SSRs) are tandemly repeated nucleotides in DNA sequences. Because of their high polymorphism, codominance and stability, SSR markers are widely used as genetic markers to analyze genetic diversity, phylogenetic relationships and the biology of populations (Sunnucks, 2000; Barkley et al., 2006). The development of SSR markers for jujube is therefore useful for reconstruction of phylogenetic relationships among cultivars, assessment of genetic diversity, and for breeding and genetic analysis of this species.

Several studies have reported the development of SSR markers for jujube. For example, 66 expressed sequence tag-SSRs were developed from a Chinese jujube fruit cDNA library and amplified in six cultivars, but without evaluation of polymorphism (Liu et al., 2014). Using selectively amplified microsatellites, Ma, Wang \& Liang (2011) constructed a set of 25 primer pairs, and an additional 31 primer pairs were developed from SSR-enriched genomic libraries (Wang et al., 2014). Seventy-one polymorphic tri-nucleotide SSR markers were developed from assembly fruit transcriptome of jujube (Li et al., 2014). A set of 551 primer pairs were developed from jujube genome and tested in 6 jujube cultivars (Xiao et al., 2015). These primer pairs were polymorphic among different jujube cultivars and were used to infer genetic relationships among major Chinese jujube cultivars, but few of these are validated at the level of jujube populations.

Nine microsatellites were validated on the level of jujube populations in the study about the genetic diversity and population structure of Z. acidojujuba, which is considered as the ancestor of cultivated jujube (Zhang et al., 2015). However, study about population structure of jujube cultivars is rare. Cultivars often have lower genetic polymorphism levels than wild species, such as peanut (Moretzsohn et al., 2004), soybean (Li et al., 2010), apple (Zhang et al., 2012), due to diversity loss during artificial selection and vegetative propagation. Markers with high polymorphism levels in jujube cultivar populations are thus still needed for genetic and evolutionary research on Chinese jujube. The recent availability of the jujube genome sequence 
(Liu et al., 2014) allows rapid identification of highly polymorphic markers for population genetics and facilitates SSR primer development.

For complementing previous data sets, we develop highly polymorphic markers in jujube cultivar populations and test them in populations as well. In this study, we examined the abundance of microsatellites with repeated unit lengths of 1-6 bp in jujube, characterized their distribution in the jujube genome, and developed 70 SSR markers from the genome sequence. After screening using a series of criteria, we finally used 20 polymorphic primer pairs to genetically characterize three jujube populations. The validated microsatellites could be used in research about the genetic structure and evolutionary history of Chinese jujube.

\section{MATERIALS AND METHODS}

\section{SSR identification and primer design}

The final assembly of Chinese jujube genome spans $437.65 \mathrm{Mb}(98.6 \%$ of the estimated size) with $321.45 \mathrm{Mb}$ anchored to the 12 pseudo-chromosomes (Liu et al., 2014). The 12 pseudochromosomes sequences were downloaded from GenBank (accession number: CM003114CM003125) and scanned using MSDB v2.4 software (http://msdb.biosv.com) to identify all perfect microsatellites (Weber, 1990). The identification criteria used for mono-, di-, tri-, tetra-, penta- and hexanucleotide repeats were default in MSDB, with a minimum of 12, 7, 5, 4, 4 and 4 repeats, respectively. After analyzing each chromosome independently, all 12 chromosomes were analyzed together. Genome sequences of Arabidopsis thaliana were downloaded from GenBank (accession number: CP002684-CP002688) and comparatively analyzed against the jujube genome.

After searching the complete jujube genome, primers were designed in PRIMER v5.0 (Clarke $\&$ Gorley, 2001) using loci with repeat numbers greater than 25 , for possible high polymorphism, and less than 100 for fitting with the scoring size standards. Microsatellites with mononucleotide repeats were removed when primer were designed. All primers were designed according to the following parameters: (1) product size from 100 to $400 \mathrm{bp}$ and (2) primer size from 18 to $22 \mathrm{bp}$ with an optimum size of $20 \mathrm{bp}$. All primers were synthesized by Tsingke Biological Technology Co. (Beijing, China). After screening using a series of criteria, parts of forward primer 5' ends were labeled with a fluorescent dye (FAM or HEX) in Tsingke Biological Technology Co.

\section{Plant materials and genomic DNA isolation}

In this study, we tested the amplification and polymorphism of all primers using three populations: two populations (17 individuals each) of jujube cultivars 'Bianhesuan' and 'Huizao' and one population (14 individuals) of Z. acidojujuba C.Y. Cheng et M.J. Liu, the ancestor of Chinese jujube (Liu \& Wang, 2009). We treated all plants of a cultivar collected from its major planting regions as a population. In addition, 10 main jujube cultivars were obtained from the Xinzheng Jujube Research Institute, Henan, China, to test the amplification feasibility of SSR markers in different cultivars. One individual was collected for each cultivar. Sample information is provided in Table S1. Total genomic DNA was extracted from dried leaves with a modified CTAB method (Doyle and Doyle, 1987), quantified using a NanoDrop 2000c 
114 spectrophotometer (Thermo Scientific, Waltham, MA, USA), and checked for quality by gel

115 electrophoresis.

116 Polymerase chain reaction (PCR) and fragment analysis

117 PCR amplifications were carried out in 15- $\mu$ l reaction volumes containing 10-20 ng template 118 DNA, $1 \times$ PCR Buffer, $1.5 \mathrm{mM} \mathrm{MgCl}_{2}, 0.4 \mu \mathrm{M}$ of each dNTP, $0.2 \mu \mathrm{M}$ of each primer and 1 unit 119 of Taq DNA polymerase (Takara, Dalian, China). The PCR cycling profile consisted of an initial 120 step of $95^{\circ} \mathrm{C}$ for $5 \mathrm{~min}$, followed by 35 cycles of $95^{\circ} \mathrm{C}$ for $45 \mathrm{~s}, 50-55^{\circ} \mathrm{C}$ (annealing temperature 121 of each primer) for $45 \mathrm{~s}$ and $72^{\circ} \mathrm{C}$ for $30 \mathrm{~s}$, with a final extension step of $72^{\circ} \mathrm{C}$ for $7 \mathrm{~min}$. The PCR products were subsequently detected using a 3730XL Genetic Analyzer Sequencer (Applied Biosystems, Foster City, California). Allele sizing was performed in GENEMAPPER v4.0 (Applied Biosystems) by comparing alleles with a GeneScan-500LIZ size standard (Applied Biosystems).

\section{Analysis of genetic diversity}

The presence of null alleles, scoring errors and large allele dropout was checked using MICROCHECKER version 2.2.3 (Van Oosterhout et al., 2004). The null allele frequency ( $r$ ), inbreeding coefficient (Fis) and linkage disequilibrium (LD) between all pairs of polymorphic loci were calculated using program GENEPOP 4.0 (Raymond \& Rousset, 1995; Rousset, 2008). The LD was tested with 10,000 permutations. The number of alleles per locus $\left(N_{a}\right)$, observed $\left(H_{o}\right)$ and expected heterozygosity $\left(H_{e}\right)$, deviations from Hardy-Weinberg equilibrium $(\mathrm{HWE})$ were calculated using ARLEQUIN version 3.5 (Excoffier \& Lischer, 2010). The HWE was tested with $1,000,000$ steps in Markov chain.

\section{RESULTS}

\section{Characterization of SSR distribution}

The frequency and density of SSRs on each of the 12 jujube chromosomes ranged from 818.29 (chromosome 5) to 920.85 (chromosome 12) loci/Mb and from 16,035.53 (chromosome 5) to $17,750.21$ (chromosome 12) bp/Mb, respectively. Analysis of $321.56 \mathrm{Mb}$ of sequence data from the assembled jujube genome uncovered 280,596 SSRs covering 5.37 Mb. These SSRs included 142,795 mono-, 87,362 di-, 29,900 tri-, 15,827 tetra-, 3,237 penta- and 1,475 hexanucleotide repeats, which respectively corresponded to $46.88 \%, 35.12 \%, 10.87 \%, 5.22 \%, 1.27 \%$ and $0.73 \%$ of total SSRs. There were 373 SSRs with more than 100 repeat motifs in the jujube genome, the longest of which comprised 2,446 AT repeats. Analysis of $119.15 \mathrm{Mb}$ of the A. thaliana genome revealed only two SSRs with more than 100 repeat motifs (201 AAG and 117 TCT). Average SSR sizes in jujube and $A$. thaliana genomes were 19.14 and $17.40 \mathrm{bp}$, respectively. Jujube genome SSR frequency and density were $872.60 \mathrm{loci} / \mathrm{Mb}$ and $16,700.42 \mathrm{bp} / \mathrm{Mb}$, respectively, with corresponding values of $221.78 \mathrm{loci} / \mathrm{Mb}$ and $3,858.19 \mathrm{bp} / \mathrm{Mb}$ in A. thaliana.

SSR frequency and density varied with motif length. As motif length increased (from monoto hexanucleotide repeats), the frequency of repeats decreased from 444.06 to 4.59 loci/Mb and density decreased from 7,829.71 to $121.64 \mathrm{bp} / \mathrm{Mb}$ (Fig. 1). The 10 most frequent motif types in the jujube genome were two mononucleotide (A/T, G/C), three dinucleotide (AT/TA, AG/CT, GT/AC), four trinucleotide (ATT/AAT, AAG/CTT, ATC/GAT, AAC/GTT) and one 
154 tetranucleotide (ATTT/AAAT) repeats (Table 1). When calculating the frequency of each motif 155 type, we considered all possible types in a repeat sequence, such as two potential types (AG and 156 GA) for the AG motif repeat and three potential types (ATT, TTA and TAT) for the ATT motif 157 repeat. As for $A$. thaliana, no tetranucleotide repeats were observed among the 10 most frequent 158 motif types. The average length, frequency and density of the 10 most frequent microsatellite motif types are provided in Table 1.

\section{Primer design and evaluation}

Taking no account of mononucleotide repeats, a total of 664 SSRs with repeat numbers greater than 25 and less than 100 were detected in jujube. We randomly selected 100 SSRs from the 664 loci and successfully designed 70 primer pairs. Screened in $2 \%$ agarose electrophoresis, 42 of the 70 primer pairs produced clear, unique amplification products of the expected size. All the 42 SSR markers showed their ability to detect polymorphisms across jujube cultivars and wild species. A set of 20 SSR markers that amplified the most easily scorable fragment polymorphisms was chosen to label with a fluorescent dye and evaluate polymorphism in the three populations. Characteristics of these 20 SSR loci are listed in Table 2. All 20 primer pairs were also amplified successfully in the 10 jujube cultivars. Among the 20 loci, $N_{a}$ ranged from 6 to 21 , with an average of 12.8 alleles per locus (Table 2). The mean value of $r$ among the three populations ranged from 0 to 0.261 . Loci Juju18 presented too many null alleles $(r>0.15)$ in all populations and was eliminated from further analysis. Average $H_{o}$ and $H_{e}$ across the three populations were 0.909 and 0.701 , respectively. The $N_{a}$ had the highest mean values (8.5) in population 1 (Z. acidojujuba) among the three populations (Table 3). Population 2 ('Bianhesuan') has the highest $H_{o}(0.967)$ but lowest $H_{e}(0.578)$. The $H_{o}$ and $H_{e}$ were zero at locus Juju64 in population 2. Of the 20 loci, 16 (all except Juju18, Juju57, Juju63, Juju64 and Juju68) showed significant deviation from HWE in two cultivar populations (Table 3). None loci showed significant deviation from HWE in population 1. The Fis of the three populations were -0.066, 0.490 and -0.305 , respectively. Three locus pairs (Juju11 \&Juju23, Juju11 \&Juju25 and Juju25 \&Juju42) showed signficant LD $(P<0.001)$ across the three populations.

\section{DISCUSSION}

\section{Characterization of SSR abundance}

The characterization of SSRs in our study was different from that reported by Xiao et al. (2015). This difference was caused by two reasons. One was that their study was based on scaffold sequences and analysis $396.18 \mathrm{Mb}$ of sequence data (Xiao et al., 2015) and our study was based on assembled 12 chromosomes and analysis $321.56 \mathrm{Mb}$ of sequence data. The other was the different identification criteria of defining SSR. Their identification criteria used for mono-, di-, tri-, tetra-, penta- and hexanucleotide repeats were a minimum of 10, 5, 5, 5, 5 and 5, respectively (Xiao et al., 2015); however, ours were a minimum of 12, 7, 5, 4, 4 and 4. We used stricter criteria for mono- and dinucleotide repeats and therefore got less SSR loci. Although the results of two studies were different, they got consistent conclusions.

We calculated the frequencies of perfect SSRs composed of 1-6-bp long motifs in jujube and A. thaliana. The abundance of certain repeat types differed between the two plants. Jujube had a 
194 much higher SSR density than A. thaliana, as well as mulberry, peach, apple, pear, grape,

195 strawberry and Prunus mume (Xiao et al., 2015). SSRs containing A and T (A/T, AT/TA and ATT/AAT) were dominant in the jujube genome, consistent with its high AT content $(66.59 \%$, Liu et al., 2014). High proportions of (A+T)-rich repeats (Xiao et al., 2015) are also present in $A$. thaliana and other plants (Lagercrantz, Ellegren \& Andersson, 1993). In protein-coding regions, the high proportion of $(\mathrm{A}+\mathrm{T})$-rich repeats is due to the poly $(\mathrm{A} / \mathrm{T})$ tails of densely scattered retroposed sequences and processed pseudogenes (Tóth, Gáspári \& Jurka, 2000).

Microsatellites are associated with nonrepetitive DNA and have their highest frequencies in transcribed regions (Morgante, Hanafey \& Powell, 2002). Besides their utility as genetic markers, SSRs have important developmental, gene regulatory and evolutionary functions (Lawson \& Zhang, 2006). The function of the high-density SSRs in the complex jujube genome (Liu et al., 2014) thus warrants further study.

The frequency of repeats in jujube decreased with increasing motif length, similar to sorghum, rice, Medicago, Populus, Brachypodium and Brassica oleracea (Iniguez-Luy, Voort \& Osborn, 2008; Sonah et al., 2011). Mononucleotide repeats were the most abundant repeats. One of the 10 most abundant motif types in jujube were tetranucleotide motifs (ATTT/AAAT) which was not found in A. thaliana. This difference may be ascribed to the complexity of the jujube genome and its high SSR density, as well as its high AT content (66.59\%, Liu et al., 2014).

\section{Primer evaluation}

More alleles were detected in population 1 than in populations 2 and 3 . This difference may be partly explained by the fact that population 1 consisted of a wild species whereas populations 2 and 3 were cultivars. It is relevant to the lower Fis in population 1 than in populations 2 and 3. Wild species often have higher genetic polymorphism levels than cultivars, such as peanut (Moretzsohn et al., 2004), soybean (Li et al., 2010), apple (Zhang et al., 2012). Using nine microsatellites, the average $H_{o}$ and $H_{e}$ of $31 \mathrm{Z}$. acidojujuba populations was 0.679 and 0.659 , respectively (Zhang et al., 2015). Comparing the Z. acidojujuba population in our study (from Luoyang) with their population from Luoyang (EYYC), our markers identified higher $N_{a}(8.5 \mathrm{vs}$ 3.9) and higher $H_{o}$ and $H_{e}$ (0.901 and 0.844 vs 0.683 and 0.625 , respectively) (Zhang et al., 2015). The higher values hinted that our marks could uncover subtler genetic structure. Locus Juju64 uncovered only one allele in population 2, causing $H_{o}$ and $H_{e}$ to be zero, and also uncovered least alleles in other two populations, thus revealing the low polymorphism of the locus.

Out of the 70 screened primer pairs, $42(60.0 \%)$ produced clear, unique amplification products and $20(28.6 \%)$ displayed high levels of polymorphism. Loci Juju18 presented too many null alleles thus was eliminated. After testing in three populations, all the remained 19 polymorphic SSRs were in HWE in at least one population. For our populations, especially population 2 and 3, many loci were not in HWE because cultivars are not in a random mating system. Taking LD into consideration, the removal of one locus (i.e., Juju11 and Juju25) from each linked pair would give 17 unlinked loci in HWE.

In conclusion, we developed 17 validated microsatellite primer pairs with applicability to jujube population genetics. These polymorphic markers will facilitate the study of jujube genetic 
235 structure and gene flow and aid investigations of the evolutionary history of this important fruit 236 crop.

\section{ACKNOWLEDGEMENT}

239 We thank Shan-shan Sun of the Wuhan Botanical Garden, Chinese Academy of Sciences, for 240 providing laboratory support. 


\section{REFERENCES}

242 Barkley NA, Roose ML, Krueger RR, Federici CT. 2006. Assessing genetic diversity and population structure in a citrus germplasm collection utilizing simple sequence repeat markers (SSRs). Theoretical and Applied Genetics 112:1519-1531 DOI 10.1007/s00122006-0255-9.

Chen Y, Schirarend C. 2007. Rhamnaceae. In: Wu ZY, Raven PH, Hong DY. Flora of China, Vol. 12. Beijing: Science Press, 115-119.

Doyle JJ, Doyle JL. 1987. A rapid DNA isolation procedure for small quantities of fresh leaf tissue. Phytochemical Bulletin 19: 11-15.

Excoffier L, Lischer HEL. 2010. Arlequin suite ver 3.5: A new series of programs to perform population genetics analyses under Linux and Windows. Molecular Ecology Resources 10: 564-567 DOI 10.1111/j.1755-0998.2010.02847.x.

Iniguez-Luy FL, Voort AV, Osborn TC. 2008. Development of a set of public SSR markers derived from genomic sequence of a rapid cycling Brassica oleracea L. genotype. Theoretical and applied genetics 117:977-985 DOI 10.1007/s00122-008-0837-9.

Lagercrantz U, Ellegren H, Andersson L. 1993. The abundance of various polymorphic microsatellite motifs differs between plants and vertebrates. Nucleic Acids Research, 21:1111-1115 DOI 10.1093/nar/21.5.1111.

Lawson MJ, Zhang LQ. 2006. Distinct patterns of SSR distribution in the Arabidopsis thaliana and rice genomes. Genome Biology 7:R14 DOI 10.1186/gb-2006-7-2-r14.

Li JW, Fan LP, Ding SD, Ding XL. 2007. Nutritional composition of five cultivars of Chinese jujube. Food Chemistry 103:454-460 DOI 10.1016/j.foodchem.2006.08.016.

Li YH, Li W, Zhang C, Yang L, Chang RZ, Gaut BS, Qiu LJ. 2010. Genetic diversity in domesticated soybean (Glycine max) and its wild progenitor (Glycine soja) for simple sequence repeat and single-nucleotide polymorphism loci. New Phytologist 188:242-253 DOI 10.1111/j.1469-8137.2010.03344.x

Li YY, Xu CQ, Lin XG, Cui BB, Wu RL, Pang X. 2014. De novo assembly and characterization of the fruit transcriptome of Chinese jujube (Ziziphus jujuba Mill.) using 454 pyrosequencing and the development of novel tri-nucleotide SSR markers. PLoS One 9:e106438 DOI 10.1371/journal.pone.0106438.

Liu, MJ. 2008. China Jujube Development Report ,1949-2007. Beijing: China Forestry Publishing.

Liu MJ, Wang M. 2009. Origin and cultivation history of jujube. In: Liu MJ, Wang M. Germplasm resources of Chinese jujube. Beijing: China Forestry Publishing, 83-85.

Liu MJ, Zhao Z. 2009. Germplasm resources and production of jujube in China. ISHS Acta Horticulturae 840:25-32 DOI 10.17660/ActaHortic.2009.840.1.

Liu MJ, Liu P, Liu GN. 2013. Advances of research on germplasm resources of Chinese jujube. Scientia Horticulturae 993:15-20 DOI 10.17660/ActaHortic.2013.993.1.

Liu J, Liu HB, Ma LY, Wang SQ, Gao J, Li YY, Wu RL, Pang XM. 2014. A Chinese jujube (Ziziphus jujuba Mill.) fruit-expressed sequence tag (EST) library: Annotation and EST- 
SSR characterization. Scientia Horticulturae 165:99-105 DOI 10.1016/j.scienta.2013.10.033.

Liu MJ, Zhao J, Cai QL, Liu GC, Wang JR, Zhao ZH, Liu P, Dai L,Yan GJ, Wang WJ, Li XS, Chen Y, Sun YD, Liu ZG, Lin MJ, Xiao J, Chen YY, Li XF, Wu B, Ma Y, Jian JB, Yang W, Yuan Z, Sun XC, Wei YL, Yu LL, Zhang C, Liao SG, He RJ, Guang XM, Wang Z, Zhang YY, Luo LH. 2014. The complex jujube genome provides insights into fruit tree biology. Nature Communication, 5:5315 DOI 10.1038/ncomms6315.

Ma QH, Wang GX, Liang LS. 2011. Development and characterization of SSR markers in Chinese jujube (Ziziphus jujuba Mill.) and its related species. Scientia Horticulturae 129:597-602 DOI 10.1016/j.scienta.2011.04.032.

Moretzsohn MC, Hopkins MS, Mitchell SE, Kresovich S, Valls JF, Ferreira ME. 2004. Genetic diversity of peanut (Arachis hypogaea L.) and its wild relatives based on the analysis of hypervariable regions of the genome. BMC Plant Biology 4:11 DOI 10.1186/1471-2229-4-11.

Morgante M, Hanafey M, Powell W. 2002. Microsatellites are preferentially associated with nonrepetitive DNA in plant genomes. Nature genetics 30:194-200 DOI 10.1038/ng822.

Pan L, Quan ZW, Hu JH, Wang GY, Liu SN, He Y, Ke WD, Ding Y. 2011. Genetic diversity and differentiation of lotus (Nelumbo nucifera) accessions assessed by simple sequence repeats. Annals of Applied Biology 159: 428-441 DOI 10.1111/j.1744-7348.2011.00509.x.

Raymond M, Rousset F. 1995. GENEPOP (version 1.2): population genetics software for exact tests and ecumenicism. Journal of Heredity 86:248-249.

Rousset F. 2008. Genepop'007: a complete reimplementation of the Genepop software for Windows and Linux. Molecular Ecology Resources 8: 103-106 DOI 10.1111/j.14718286.2007.01931.x.

Sefc KM, Lopes MS, Lefort F, Botta R, Roubelakis-Angelakis KA, Ibáñez J, Pejić I, Wagner HW, Glössl J, Steinkellner H. 2000. Microsatellite variability in grapevine cultivars from different European regions and evaluation of assignment testing to assess the geographic origin of cultivars. Theoretical and Applied Genetics 100: 498-505 DOI $10.1007 / \mathrm{s} 001220050065$.

Sonah H, Deshmukh RK, Sharma A, Singh VP, Gupta DK, Gacche RN, Rana JC, Singh NK, Sharma TR. 2011. Genome-wide distribution and organization of microsatellites in plants: an insight into marker development in Brachypodium. Plos one 6:e21298 DOI 10.1371/journal.pone.0021298.

Sunnucks P. 2000. Efficient genetic markers for population biology. Trends in Ecology \& Evolution 15:199-203 DOI 10.1016/S0169-5347(00)01825-5.

Testolin R, Marrazzo T, Cipriani G, Quarta R, Verde I, Dettori MT, Pancaldi M, Sansavini S. 2000. Microsatellite DNA in peach (Prunus persica L. Batsch) and its use in fingerprinting and testing the genetic origin of cultivars. Genome 43: 512-520 DOI: 10.1139/g00-010.

Tóth G, Gáspári Z, Jurka J. 2000. Microsatellites in different eukaryotic genomes: survey and 
analysis. Genome research 10:967-981 DOI 10.1101/gr.10.7.967.

Van Oosterhout C, Hutchinson WF, Wills DPM, Shipley P. 2004. Micro-Checker: software for identifying and correcting genotyping errors in microsatellite data. Molecular Ecology Notes 4:535-538 DOI 10.1111/j.1471-8286.2004.00684.x.

Wang YL. 2001. Prospects of extension for propagation techniques for green cuttages in Jujube. Economic Forest Researches 9:59-60 (in Chinese).

Wang SQ, Liu Ying, Ma LY, Liu HB, Tang Yan, Wu LP, Wang Z, Li YY, Wu RL, Pang XM. 2014. Isolation and Characterization of Microsatellite Markers and Analysis of Genetic Diversity in Chinese Jujube (Ziziphus jujuba Mill.), PLoS ONE 9:e99842 DOI 10.1371/journal.pone.0099842.

Weber JL. 1990. Informativeness of human $(\mathrm{dC}-\mathrm{dA}) \mathrm{n} \cdot(\mathrm{dG}-\mathrm{dT}) n$ polymorphisms. Genomics 7:524-530 DOI 10.1016/0888-7543(90)90195-Z.

Xiao J, Zhao J, Liu MJ, Liu P, Dai L, Zhao ZH. 2015. Genome-wide characterization of simple sequence repeat (SSR) loci in Chinese jujube and jujube SSR primer transferability. PLoS One 10: e0127812 DOI 10.1371/journal.pone.0127812.

Zhang Q, Li J, Zhao Y, Korban SS, Han Y. 2012. Evaluation of genetic diversity in Chinese wild apple species along with apple cultivars using SSR markers. Plant molecular biology reporter 30: 539-546 DOI 10.1007/s11105-011-0366-6.

Zhang CM, Huang J, Yin X, Lian CL, Li XG. 2015. Genetic diversity and population structure of sour jujube, Ziziphus acidojujuba. Tree Genetics \& Genomes 11: 809 DOI 10.1007/s11295-014-0809-y. 


\section{1}

The density $(\mathrm{bp} / \mathrm{Mb})$ of microsatellite with repeated unit lengths of 1-6 base pairs in genome of Chinese jujube (Ziziphus jujuba Mill) and Arabidopsis thaliana.

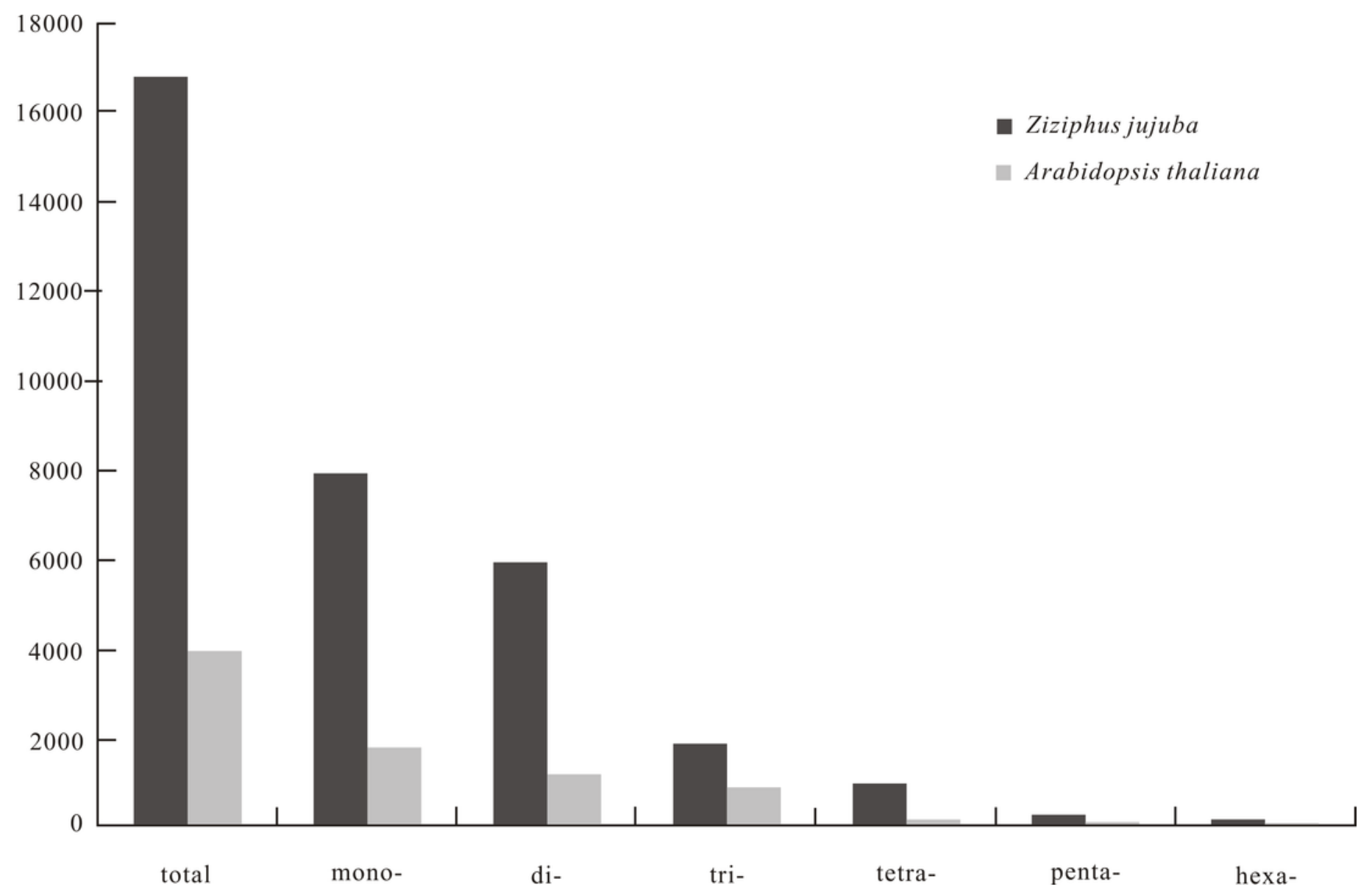




\section{Table $\mathbf{1}$ (on next page)}

The ten most frequency microsatellite motif type in genome of Chinese jujube (Ziziphus jujuba Mill) and Arabidopsis thaliana. 


\begin{tabular}{rllrrrrrr}
\hline \multirow{2}{*}{ No. } & \multicolumn{3}{c}{ Motif } & \multicolumn{2}{c}{ Average Length (bp) } & \multicolumn{2}{c}{ Frequency (loci/Mb) } & \multicolumn{2}{c}{ Density (bp/Mb) } \\
\cline { 2 - 9 } & Z. jujuba & A. thaliana & Z. jujuba & A. thaliana & Z. jujuba & A. thaliana & Z. jujuba & A. thaliana \\
\hline 1 & A/T & A/T & 17.64 & 14.98 & 436.39 & 112.63 & 7699.69 & 1686.89 \\
2 & AT/TA & AT/TA & 20.37 & 22.60 & 201.50 & 30.68 & 4103.91 & 693.38 \\
3 & ATT/AAT & AAG/CTT & 19.59 & 18.63 & 59.10 & 21.13 & 1157.87 & 393.79 \\
4 & AG/CT & AG/CT & 25.04 & 20.86 & 50.39 & 16.95 & 1261.91 & 353.66 \\
5 & ATTT/AAAT & ATG/CAT & 17.54 & 18.51 & 39.07 & 7.34 & 685.46 & 135.76 \\
6 & GT/AC & AAC/GTT & 24.39 & 17.96 & 19.73 & 5.81 & 481.22 & 104.31 \\
7 & AAG/CTT & GT/AC & 20.07 & 16.72 & 17.72 & 4.30 & 355.69 & 71.84 \\
8 & G/C & AAT/ATT & 16.94 & 19.24 & 7.68 & 3.31 & 130.12 & 63.63 \\
9 & ATC/GAT & AGG/CCT & 19.19 & 17.19 & 5.16 & 2.93 & 99.02 & 50.36 \\
10 & AAC/GTT & ACC/GGT & 18.90 & 16.80 & 5.05 & 1.99 & 95.52 & 33.41 \\
\hline
\end{tabular}

1 Note: Each motif type contained all the possible cases in a repeat sequence; for example, AG contained AG and GA, ATT contained ATT, TTA and TAT. 


\section{Table 2 (on next page)}

Characteristics of 20 microsatellite loci and primer pairs developed from Chinese jujube (Ziziphus jujuba Mill) genome. 


\begin{tabular}{|c|c|c|c|c|c|c|c|c|}
\hline Locus & Primer sequences $\left(5^{\prime}-3^{\prime}\right)$ & Repeat motif & Size (bp) & $\mathrm{Ta}\left({ }^{0} \mathrm{C}\right)$ & $\begin{array}{l}\text { Total no. } \\
\text { of alleles }\end{array}$ & $\begin{array}{c}\text { Fluorescent } \\
\text { dye }\end{array}$ & Location & $\begin{array}{c}\text { Accession in } \\
\text { Genebank }\end{array}$ \\
\hline Juju2 & F: ACATGGAGAAATGGGATC & $(\mathrm{AG})_{87}$ & $220-256$ & 53 & 15 & FAM & add_scaffold 107 & KN813357.1 \\
\hline \multirow[t]{2}{*}{ Juju5 } & F: GGCGACGATTAGAGGAAA & $(\mathrm{AG})_{36}$ & $202-226$ & 53 & 7 & FAM & add_scaffold 247 & KN813805.1 \\
\hline & R: CTGCTTGTACGGCCAGTT & & & & & & & \\
\hline \multirow[t]{2}{*}{ Juju11 } & F: CCACTTGCGTTACTTCTC & $(\mathrm{CT})_{92}$ & $192-222$ & 53 & 8 & FAM & chromosome 6 & CM003119.1 \\
\hline & R: AATCTCGCTTTGCTCTAT & & & & & & & \\
\hline \multirow[t]{2}{*}{ Juju13 } & F: TAGGCATTTGCATGGTAT & $(\mathrm{TC})_{32}$ & $190-208$ & 53 & 8 & HEX & chromosome 3 & CM003116.1 \\
\hline & R: TTGTCCGCTTTCTTGAGT & & & & & & & \\
\hline \multirow[t]{2}{*}{ Juju23 } & F: CCATCCGACCACTGAAAT & $(\mathrm{AG})_{29}$ & $107-163$ & 53 & 20 & FAM & chromosome 10 & CM003123.1 \\
\hline & R: CGTAAAGCACCAGCAAAA & & & & & & & \\
\hline \multirow[t]{2}{*}{ Juju25 } & F: GTACGGTATGACTCCACA & $(\mathrm{CA})_{48}$ & $116-192$ & 53 & 17 & FAM & chromosome 3 & CM003116.1 \\
\hline & R: CATCCAATCACTGAAAAT & & & & & & & \\
\hline \multirow[t]{2}{*}{ Juju30 } & F: AAATGACCATCGAATCCC & $(\mathrm{GA})_{25}$ & $224-260$ & 53 & 11 & HEX & chromosome 11 & CM003124.1 \\
\hline & R: CTTTGTTGTTACCCCAGA & & & & & & & \\
\hline \multirow[t]{2}{*}{ Juju35 } & F: TTGGATTAGTGTACTTGG & $(\mathrm{TC})_{47}$ & $109-219$ & 53 & 12 & HEX & chromosome 6 & CM003119.1 \\
\hline & R: ACATGAGGAAACCTGGAA & & & & & & & \\
\hline Juju42 & F: CTTCAGGACGGACCAAAT & $(\mathrm{AG})_{25}$ & $150-230$ & 50 & 21 & HEX & chromosome 8 & CM003121.1 \\
\hline
\end{tabular}




\section{R: AACTGATCCTCCTTCGTC}

Juju52 F: TTGAAAAGGAAGGAAGAG R: TGAGGATTATGAAGGGTT

Juju54 F: GAATCCTTACATCCAATA R: ACTTACCATAATCTGTGC

Juju57 F: ATTTATTCCTTATTGCTAGTAG R: CAACCTTCTTGTAGTTATTTT

Juju63 F: ATCAGCCAGCGTCACAAA R: ATCCAAATAAGCCCACCT

Juju64 F: ATATTGGAAACTTTCTGATC R: CTGTAATACTGGGATGCT

Juju66 F: TGGATACCGTGAAGGAAC R: AGCCCATTAGAAAGCAAC

Juju68 F: AGGCTTCAACTCTTATCC R: CCAAAACCACCACAAAAT

\begin{tabular}{|c|c|c|c|c|c|c|}
\hline$(\mathrm{TG})_{28}$ & 203-229 & 50 & 10 & HEX & chromosome 6 & CM003119.1 \\
\hline$(\mathrm{TACA})_{37}$ & $86-158$ & 53 & 6 & HEX & chromosome 4 & CM003117.1 \\
\hline$(\mathrm{CA})_{27}$ & $122-210$ & 55 & 18 & HEX & scaffold 844 & KN812877.1 \\
\hline$(\mathrm{TA})_{35}$ & $155-205$ & 55 & 10 & FAM & chromosome 11 & CM003124.1 \\
\hline$(\mathrm{TC})_{97}$ & $108-138$ & 53 & 6 & FAM & chromosome 11 & CM003124.1 \\
\hline$(\mathrm{GA})_{68}$ & $178-216$ & 53 & 13 & HEX & chromosome 7 & CM003120.1 \\
\hline$(\mathrm{TAA})_{61}$ & $128-204$ & 53 & 19 & FAM & chromosome 1 & CM003114.1 \\
\hline
\end{tabular}

Note: $T \mathrm{a}=$ annealing temperatur 


\section{Table 3(on next page)}

Results of initial primer screening in three populations.

One population is Ziziphus. acidojujuba C.Y. Cheng et M.J. Liu and the other two are cultivars "Bianhesuan" and "Huizao". 


\begin{tabular}{|c|c|c|c|c|c|c|c|c|c|c|c|c|c|c|c|}
\hline \multirow{2}{*}{ Locus } & \multicolumn{5}{|c|}{ Z. acidojujuba $(\mathrm{N}=14)$} & \multicolumn{5}{|c|}{ 'Bianhesuan' ( $\mathrm{N}=17)$} & \multicolumn{5}{|c|}{ 'Huizao' (N = 17) } \\
\hline & $\mathrm{Na}$ & $r$ & $\mathrm{H}_{o}$ & $\mathrm{H}_{e^{\mathrm{a}}}$ & Fis & $\mathrm{Na}$ & $r$ & $\mathrm{H}_{o}$ & $\mathrm{H}_{e^{\mathrm{a}}}$ & Fis & $\mathrm{Na}_{\mathrm{a}}$ & $r$ & $\mathrm{H}_{o}$ & $\mathrm{H}_{e^{\mathrm{a}}}$ & Fis \\
\hline Juju2 & 10 & 0.000 & 1.000 & 0.846 & -0.182 & 6 & 0.039 & 1.000 & $0.649 *$ & -0.568 & 3 & 0.298 & 0.727 & $0.685^{*}$ & -0.074 \\
\hline Juju5 & 6 & 0.000 & 0.857 & 0.757 & -0.139 & 3 & 0.000 & 0.941 & $0.544^{*}$ & -0.772 & 3 & 0.000 & 0.667 & $0.665^{*}$ & 0.059 \\
\hline Juju10 & 6 & 0.000 & 0.800 & 0.728 & -0.059 & 7 & 0.000 & 0.941 & $0.695^{*}$ & -0.369 & 6 & 0.231 & 0.455 & $0.749^{*}$ & 0.444 \\
\hline Juju11 & 6 & 0.000 & 0.875 & 0.827 & -0.077 & 3 & 0.000 & 1.000 & $0.569 *$ & -0.801 & 4 & 0.051 & 1.000 & $0.720^{*}$ & -0.345 \\
\hline Juju13 & 6 & 0.076 & 0.714 & 0.765 & 0.068 & 5 & 0.000 & 1.000 & 0.386 & 0.000 & 5 & 0.139 & 0.538 & $0.706^{*}$ & 0.276 \\
\hline Juju17 & 6 & 0.000 & 0.769 & 0.783 & 0.036 & 4 & 0.000 & 1.000 & 0.298 & 0.000 & 6 & 0.000 & 1.000 & $0.619^{*}$ & -0.649 \\
\hline Juju18 & 10 & 0.137 & 1.000 & 0.902 & -0.113 & 4 & 0.400 & 0.667 & 0.363 & 0.273 & 7 & 0.246 & 1.000 & 0.820 & -0.228 \\
\hline Juju23 & 12 & 0.000 & 1.000 & 0.913 & -0.100 & 3 & 0.000 & 1.000 & $0.544^{*}$ & -0.889 & 4 & 0.000 & 1.000 & $0.697 *$ & -0.455 \\
\hline Juju25 & 11 & 0.000 & 0.786 & 0.884 & 0.115 & 4 & 0.000 & 1.000 & $0.644^{*}$ & -0.581 & 11 & 0.076 & 0.765 & $0.868^{*}$ & 0.122 \\
\hline Juju30 & 10 & 0.000 & 1.000 & 0.889 & -0.116 & 4 & 0.000 & 1.000 & $0.622 *$ & -0.639 & 3 & 0.000 & 0.909 & 0.500 & -0.681 \\
\hline Juju35 & 7 & 0.159 & 0.929 & 0.857 & -0.087 & 3 & 0.000 & 1.000 & $0.544 *$ & -0.889 & 3 & 0.161 & 1.000 & $0.544^{*}$ & -0.889 \\
\hline Juju42 & 12 & 0.037 & 0.857 & 0.915 & 0.066 & 7 & 0.000 & 1.000 & $0.647^{*}$ & -0.572 & 11 & 0.060 & 0.824 & $0.854^{*}$ & 0.037 \\
\hline Juju43 & 8 & 0.000 & 1.000 & 0.863 & -0.154 & 5 & 0.000 & 0.882 & $0.642 *$ & -0.391 & 5 & 0.000 & 1.000 & 0.748 & -0.360 \\
\hline Juju52 & 10 & 0.000 & 1.000 & 0.913 & -0.100 & 6 & 0.000 & 0.941 & $0.759 *$ & -0.249 & 3 & 0.035 & 0.647 & $0.611^{*}$ & -0.060 \\
\hline Juju54 & 5 & 0.000 & 0.857 & 0.754 & -0.143 & 4 & 0.000 & 1.000 & $0.571 *$ & -0.795 & 2 & 0.000 & 0.938 & $0.511^{*}$ & -0.875 \\
\hline Juju57 & 11 & 0.000 & 1.000 & 0.909 & -0.125 & 5 & 0.000 & 1.000 & 0.609 & -0.395 & 9 & 0.055 & 0.833 & 0.778 & -0.084 \\
\hline Juju63 & 7 & 0.100 & 0.900 & 0.844 & -0.066 & 5 & 0.000 & 1.000 & 0.646 & -0.440 & 7 & 0.000 & 0.929 & 0.802 & -0.134 \\
\hline Juju64 & 5 & 0.000 & 0.917 & 0.726 & -0.228 & 1 & 0.000 & 0.000 & 0.000 & - & 2 & 0.000 & 1.000 & 0.453 & -1.000 \\
\hline Juju66 & 11 & 0.000 & 0.833 & 0.905 & 0.091 & 5 & 0.000 & 1.000 & $0.595 *$ & -0.716 & 2 & 0.000 & 1.000 & $0.515^{*}$ & -1.000 \\
\hline Juju68 & 11 & 0.060 & 0.917 & 0.895 & -0.017 & 5 & 0.000 & 1.000 & 0.648 & -0.507 & 6 & 0.048 & 0.923 & 0.779 & -0.205 \\
\hline Mean & 8.5 & 0.028 & 0.901 & 0.844 & -0.066 & 4.6 & 0.022 & 0.967 & 0.578 & -0.490 & 5.1 & 0.070 & 0.858 & 0.681 & -0.305 \\
\hline
\end{tabular}

1 Note: $N_{a}=$ total number of alleles per locus; $H_{o}=$ observed expected heterozygosities; $H_{e}=$ expected heterozygosities; $\mathrm{N}=$ sample size for each population; $r=$

2 null allele frequency; Fis =inbreeding coefficient. ${ }^{a}$ Significant departure from $\mathrm{HWE}$ at $* \mathrm{P}<0.01$, respectively. 